\title{
THE SOLAR JULIA SETS OF BASIC QUADRATIC CREMER POLYNOMIALS
}

\author{
A. BLOKH, X. BUfF, A. CHÉRITAT, AND L. OVERSTEEGEN
}

\begin{abstract}
In general, little is known about the exact topological structure of Julia sets containing a Cremer point. In this paper we show that there exist quadratic Cremer Julia sets of positive area such that for a full Lebesgue measure set of angles the impressions are degenerate, the Julia set is connected im kleinen at the landing points of these rays, and these points are contained in no other impression.
\end{abstract}

\section{INTRODUCTION}

A long outstanding problem of whether polynomial Julia sets of positive area exist has recently been solved in [BC06]. In that paper the authors implemented a program initiated by A. Douady and showed that there exist quadratic polynomials of various types all of which have Julia sets of positive area. The proofs are based on McMullen's results McM98 regarding the measurable density of the filled-in Julia set near the boundary of a Siegel disk with bounded type rotation number, Chéritat's techniques of parabolic explosion [C01] and Yoccoz's renormalization techniques [Yoc95] to control the shape of Siegel disks, and Inou and Shishikura's results [IS] to control the post-critical sets of perturbations of polynomials having an indifferent fixed point.

In particular, there exist quadratic polynomials with a fixed Cremer point whose Julia set has positive area. The topological structure of Julia sets containing a Cremer point (including the above mentioned ones of positive area) has remained elusive. In the present paper we want to shed some light upon this problem.

Let us first discuss the topological structure of connected polynomial Julia sets in general. The best case scenario is when the Julia set $J$

Date: June 11, 2007; the revised version October 14, 2008.

2000 Mathematics Subject Classification. Primary 37F10; Secondary 37B45, $37 \mathrm{C} 25$.

Key words and phrases. Complex dynamics; Julia set; Cremer fixed point.

The first author was partially supported by NSF grant DMS-0456748.

The fourth author was partially supported by NSF grant DMS-0405774. 
is locally connected. Then $J$ is homeomorphic to the quotient space $S^{1} / \sim=J_{\sim}$ of the unit circle with respect to a specific equivalence relation $\sim$ called an invariant lamination. Moreover, the factor map in this case conjugates the polynomial to the map $f_{\sim}$ induced on $J_{\sim}$ by the map $z^{d}: S^{1} \rightarrow S^{1}$ with $d$ being the degree of the polynomial. Spaces like $J_{\sim}$ are called below topological Julia sets while the induced maps $f_{\sim}$ on $J_{\sim}$ are called topological polynomials. Thus, in the locally connected case topological polynomials are good (one-to-one) models for true complex polynomials on their Julia sets.

The picture is more complicated when $J$ is not locally connected. Still even in that case recently developed tools allow one to use topological polynomials as models (albeit not as good as above). Given a polynomial $P$, denote by $J_{P}$ its Julia set. Call irrational neutral periodic points CS-points; a CS-point $p$ is said to be a Cremer point if the iterate of the map which fixes $p$ is not linearizable in a small neighborhood of $p$. Suppose that $P$ is a polynomial with connected Julia set and no CS-points. In his fundamental paper [K04] Jan Kiwi introduced for $P$ an invariant lamination $\sim_{P}$ on $S^{1}$ such that $\left.P\right|_{J_{P}}$ is semi-conjugate to the induced map $f_{\sim_{P}}: J_{\sim_{P}} \rightarrow J_{\sim_{P}}$ by a monotone map $m: J_{P} \rightarrow J_{\sim_{P}}$ (by monotone we mean a continuous map whose point preimages are connected). Then $J_{\sim_{P}}$ is a locally connected model for $J$, and $\left.P\right|_{J}$ is monotonically semiconjugate to $f_{\sim_{P}}$. In addition Kiwi proved in [K04] that at all periodic points $p$ of $P$ in $J_{P}$ the set $J_{P}$ is locally connected at $p$ and $m^{-1} \circ m(p)=\{p\}$. However in some cases the entire approach which uses modeling of the Julia set by means of a monotone map onto a locally connected continuum breaks down. From now on we will consider only quadratic polynomials; the critical point will be always denoted by $c$.

A quadratic polynomial $P$ with a Cremer fixed point (i.e. with a neutral non-linearizable fixed point $p \in J$ such that $P^{\prime}(p)=e^{2 \pi i \alpha}$ with $\alpha$ irrational) is said to be a basic Cremer polynomial, and its Julia set is called a basic Cremer Julia set (in this case we always denote the Cremer fixed point of $P$ by $p$ ). The main result of a recent preprint [BO06b] is that if $P$ is a basic Cremer polynomial then any monotone map $m: J_{P} \rightarrow A$ with $m\left(J_{P}\right)$ locally connected must collapse all of $J_{P}$ to a point. Hence studying the topology of basic Cremer Julia sets requires new tools (see, e.g., the fundamental papers [Per94 and Per97] by Perez-Marco); some tools here are provided by continuum theory and developed in [GMO99] (in which results of [Yoc95] were used) and further in BO06a. Before introducing them let us have an overview of a few known results. 
By Sullivan Sul83, a basic Cremer Julia set $J$ is not locally connected. Moreover, by Kiwi K00 the critical point $c$ is not accessible. Still, there are points in $J$ at which rays are landing (e.g., repelling periodic points [DH85]), so it makes sense to study in more detail the pattern in which such landing can occur. In this respect the following important question is due to C. McMullen [McM94]: can a basic Cremer Julia set contain any points at which at least two rays are landing (so-called biaccessible points)? This question was partially answered by Schleicher and Zakeri in [SZ99, Theorem 3] (see also [Zak00, Theorem 3]) where they show that if a basic Cremer Julia set contains a biaccessible point then this point eventually maps to the Cremer point. In BO06a it is shown that if a basic Cremer Julia set has a biaccessible point then it is a solar Julia set as described in Theorem 1.1. However it is still unknown if there exist basic Cremer Julia sets with biaccessible points. Another paper studying the topology of basic Cremer Julia sets is that of Sørensen [Sor98]. In that paper the author constructs basic Cremer polynomials with external rays which accumulate on both the Cremer point and its preimage and thus gives examples of basic Cremer polynomials whose Julia sets have very interesting topological properties.

Now we would like to state the results of [BO06a]. If $P$ is a basic Cremer polynomial then by [Mn93] $p \in \omega(c)$ (see also [Per97] and [C05, Theorem 1.3]). Following Kiwi [K04] we say that two angles $\theta, \gamma$ are $K$-equivalent if there are angles $x_{0}=\theta, \ldots, x_{n}=\gamma$ such that the impressions of $x_{i-1}$ and $x_{i}$ are non-disjoint for $1 \leq i \leq n$; a class of $\mathrm{K}$-equivalence is called a $K$-class, and an angle whose impression is disjoint from all other impressions is said to be K-separate. A continuum $X$ is connected im kleinen at a point $x$ provided for each open set $U$ containing $x$ there exists a connected set $C \subset U$ such that $x$ is in the interior of $C$ (relative to $X$ ). A continuum $X$ is locally connected at a point $x$ provided there exists a basis of open connected neighborhoods at $x$. Observe that sometimes different terminology is used. For example, in Milnor's book [Mil00, p. 168] the property of local connectivity is called "open local connectivity" while to the property of being connected im kleinen at a point Milnor refers to as the property of being "locally connected at a point". On the other hand, in the textbook by Munkres [Mun00, p. 162] connected im kleinen is called "weakly locally connected". Using our terminology, if a space is locally connected at $x$, then it is connected im kleinen at $x$. It is well known that if a continuum is connected im kleinen at each point, then it is locally connected (see, e.g., Mun00, p. 162, Ex. 6]). However, a continuum can be connected im kleinen at a point without being locally connected at 
this point (as an example one can consider the so-called infinite broom, see [Mun00, p. 162, Ex. 7]).

The main result of [BO06a] is the following theorem (by a degenerate impression we mean an impression consisting of one point).

Theorem 1.1. Let $P$ be a basic Cremer polynomial. Then its Julia set $J$ must be one of the following two types.

Solar Julia set: $J$ has the following equivalent properties:

(1) there is an impression not containing the Cremer point;

(2) there is a degenerate impression;

(3) the set $Y$ of all $K$-separate angles with degenerate impressions contains all angles with dense orbits ( $Y$ contains a full Lebesgue measure $G_{\delta}$-set dense in $S^{1}$ ) and a dense in $S^{1}$ set of periodic angles, and the Julia set $J$ is connected im kleinen at the landing points of these rays;

(4) there is a point at which the Julia set is connected im kleinen;

(5) not all angles are K-equivalent.

Red dwarf Julia set: $J$ has the following equivalent properties:

(1) All impressions are non-degenerate.

(2) The intersection of all impressions is a non-degenerate subcontinuum of $J$ containing the Cremer point and the limit set of the critical point.

(3) $J$ is nowhere connected im kleinen.

The main aim of this paper is to prove the following theorem.

Theorem 1.2. There exist basic Cremer polynomials with solar Julia sets of positive area.

We prove Theorem 1.2 combining results from [BC06] and [BO06a]. An interesting remaining problem then is that of the existence of red dwarf Julia sets. It is related to a well-known problem concerning the existence of indecomposable Julia sets (a continuum is indecomposable if it cannot be represented as the union of two proper subcontinua). It is known that if the Julia set is indecomposable then every impression coincides with the entire Julia set (see, e.g., [MR93] and [CMR05]).

\section{Preliminaries}

2.1. General facts and notation. In what follows we use standard tools of Carathéodory theory. An unshielded continuum $X \subset \mathbb{C}$ is a continuum which coincides with the boundary of the infinite complementary component $U$ of $X$ in the complex sphere. Given an unshielded 
continuum $X$ let $\phi$ be the normalized Riemann map from the unit disk $\mathbb{D}$ onto $U$. The external ray $R_{\alpha}$ is the image of the radius of $\mathbb{D}$ corresponding to the external angle $\alpha$. The impression $\operatorname{Imp}(\alpha)$ of $R_{\alpha}$ is defined (see, e.g., Pom92]) as the set of all limit points of sequences $\phi\left(x_{i}\right)$ taken over all sequences $x_{i} \rightarrow e^{2 \pi i \alpha}, x_{i} \in \mathbb{D}$ (if we do not want to specify the angle we will omit it from the notation). By a component of a set we always mean a connected component of this set, and by a non-separating planar set we mean a set whose complement in the plane is connected. Also, given sets $A, B$ such that $A \cap B$ is a singleton, we shall say that $A$ is attached to $B$.

Given a polynomial $f: \mathbb{C} \rightarrow \mathbb{C}$ with non-separating Julia set $J(P)$ and a continuum $K \subset J(P)$, a set $K^{\prime \prime}$ is said to be a $K$-pullback (of order $n$ by $f$ ) if $K^{\prime \prime}$ is a component of $f^{-n}(K)$. Clearly, any $K$ pullback $K^{\prime \prime}$ maps onto $K$ as a branched covering map. For us the most interesting is the case of quadratic polynomials with non-separating Julia sets and subcontinua $K$ of the Julia set. Then if $K$ contains $f(c)$, the first $K$-pullback is unique and maps onto $K$ in a 2-to- 1 branched covering fashion while if $K$ does not contain $f(c)$ then there are two first $K$-pullbacks each of which maps onto $K$ homeomorphically. Given a quadratic polynomial $f$ with the critical point $c_{f}=c$, we denote by $\psi_{f}=\psi$ the involution which maps any $z$ to the other preimage of $f(z)$ (e.g., $\psi(c)=c$, and if $f(z)=z^{2}+v$ then $\psi(z)=-z$ ). Below we often consider forward invariant continua $K \subset J_{f}$ which contain $c$ but are such that the first $n$-segment of the orbit of $c$ avoids $\psi(K)=K^{\prime}$ (i.e., $\left.f(c) \notin K^{\prime}, \ldots, f^{n}(c) \notin K^{\prime}\right)$. In this case we consider $K^{\prime}$-pullbacks of order at most $n$ ( $K^{\prime}$ is considered its own pullback of order 0$)$. Because of the assumptions, all these $K^{\prime}$-pullbacks map univalently onto $K^{\prime}$.

2.2. Siegel polynomials. A quadratic polynomial is said to be a basic Siegel polynomial if it has an invariant Siegel disk. Denote by $\mathcal{S}_{l c}$ the family of basic Siegel polynomials with locally connected Julia set. We need a few well-known facts concerning their Julia sets $J$ (see, e.g., GMO99]). Given a polynomial $P \in \mathcal{S}_{l c}$, let $\Delta$ be its closed Siegel disk, $S=\partial \Delta$, and $\psi(\Delta)=\Delta^{\prime}$. Since $J$ is locally connected, $\{c\}=$ $\Delta^{\prime} \cap \Delta$, and so $\Delta^{\prime}$ is attached to $\Delta$ at $c$ and contains no forward images of $c$. Consider the branch $c_{-1}, c_{-2}, \ldots$ of the backward orbit of $c=c_{0}$ consisting only of points of $S$ (here $\left.P\left(c_{-n-1}\right)=c_{-n}\right)$. At each point $c_{-n}$ the appropriate $n$-th pullback of $\Delta^{\prime}$, corresponding to $c_{-n}$ as the pullback of $c$, is attached to $\Delta$. However all other $\Delta^{\prime}$-pullbacks are disjoint from $\Delta$. Since all forward images of $c$ avoid $\Delta^{\prime}$ then the picture described in the previous subsection applies to $\Delta$ and we have a family of well-defined univalent $\Delta^{\prime}$-pullbacks of all orders. The entire 


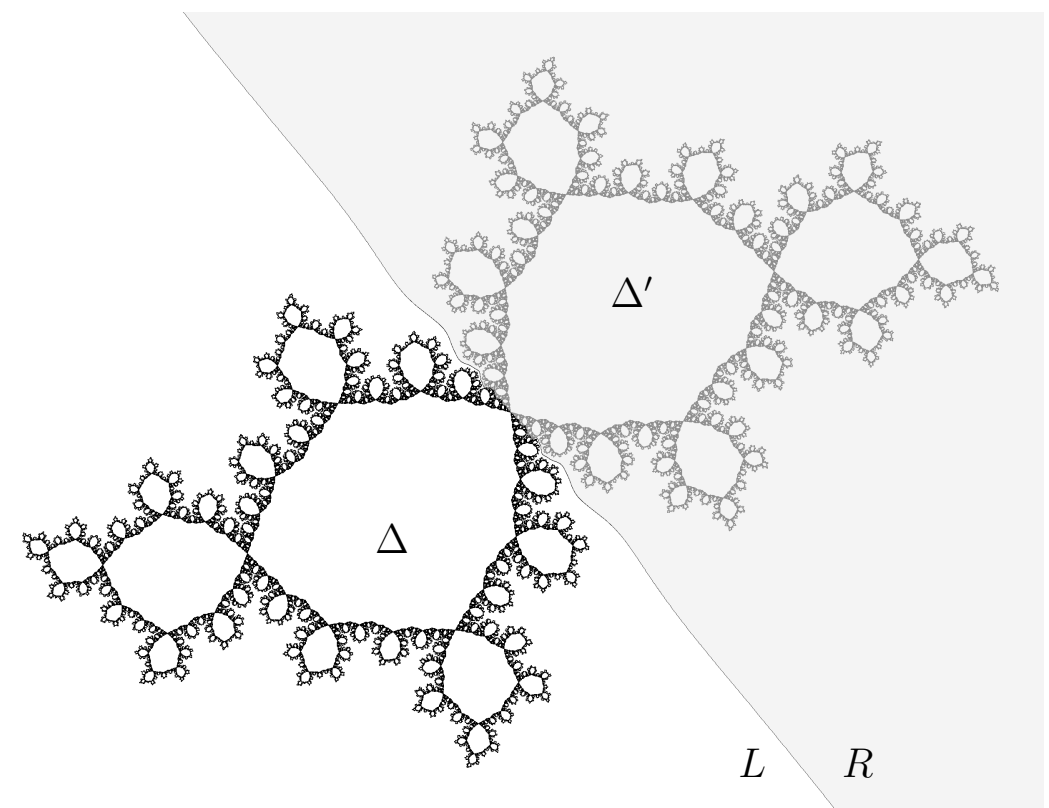

Figure 1. Example of a locally connected basic Siegel polynomial Julia set: that with the golden mean rotation number. We put the two external rays landing at the critical point and grayed the half-plane $R$.

set $P^{-n}(\Delta)$ is a connected union of $\Delta$ and $2^{n}-1 \Delta^{\prime}$-pullbacks, $n$ of which are attached to $\Delta$, while others are disjoint from $\Delta$.

The way $\Delta$ and $\Delta^{\prime}$-pullbacks intersect (but not their relative location on the plane!) is the same for all polynomials $P \in \mathcal{S}_{l c}$. To describe it, observe that two external rays landing at $c$ cut $\mathbb{C}$ into two half-planes. Assign 1 to the closed half-plane $L \supset \Delta$ and 0 to the closed half-plane $R \supset \Delta^{\prime}$ and study the symbolic dynamics of points of $J$ in terms of this partition of the plane. To each point $x \in J$ we associate its infinite itinerary $i(x)$ defined in the obvious way. Clearly, the only ambiguity in $i(x)$ arises if the point $x$ is a critical preimage because the only point of $J$ which belongs to both $L$ and $R$ is $c$ (and hence $c$ can be assigned both 0 and 1 as the first entry in its itinerary). This ambiguity is resolved though if instead of points we deal with $\Delta^{\prime}$-pullbacks. Namely, if $Q$ is $\Delta$ or a $\Delta^{\prime}$-pullback then we assign as its infinite itinerary $i(Q)$ the itinerary of any non-precritical point of $Q$ (any such itinerary $i(Q)$ from some time on consists of 1's). To simplify the notation, let us denote by $1_{i}$ the string of 1 's of length $i$ (possibly, $i=\infty$ ). Similarly, if a finite string $i^{\prime}=i_{0} i_{1} \ldots i_{l}$ is given then $i_{k}^{\prime}=\left\{i_{0} \ldots i_{l}\right\}_{k}$ is the concatenation 


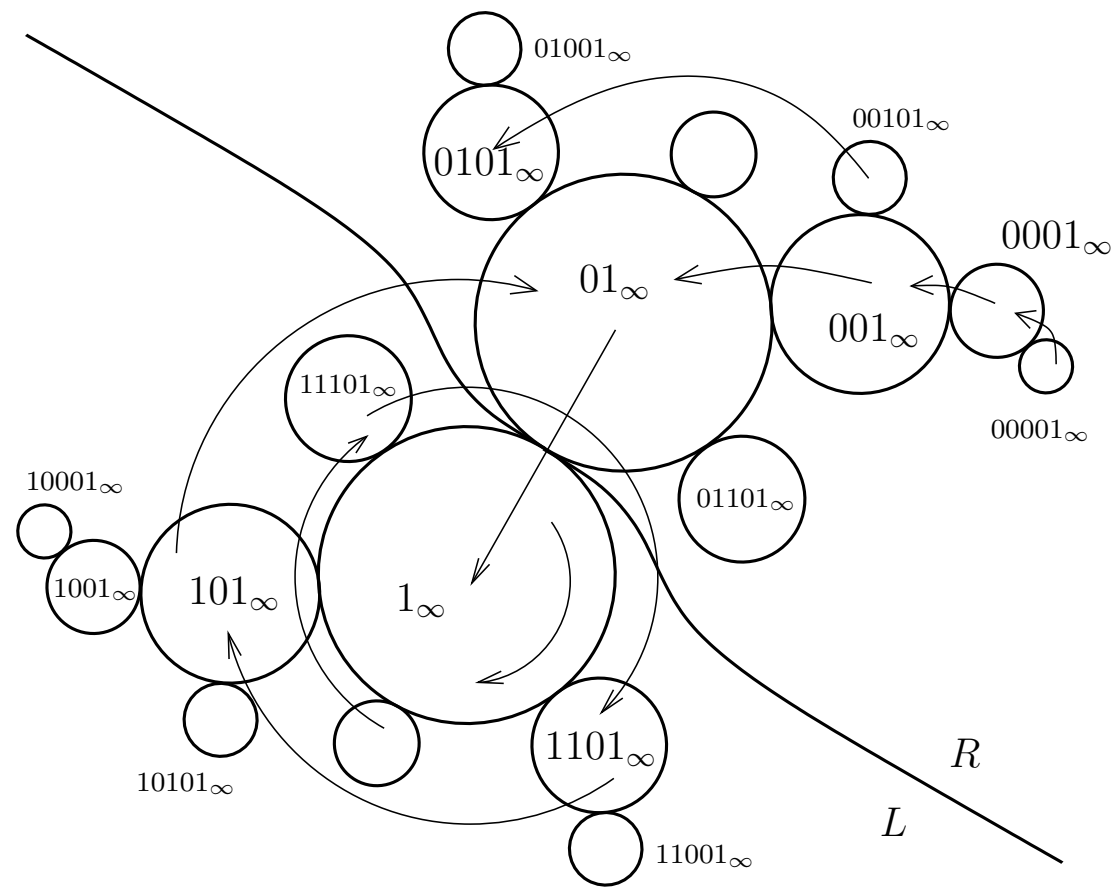

Figure 2. Example of itineraries of $\Delta$ and of pullbacks of $\Delta^{\prime}$ and of their dynamics for the Golden mean basic Siegel Julia set. Some of the missing arrows can be determined by using the central symmetry: two symmetric components are mapped to the same component.

of $k$ copies of $i^{\prime}$ (here again $k$ can be $\infty$ ). Then we have, e.g., that $i(\Delta)=1_{\infty}, i\left(\Delta^{\prime}\right)=01_{\infty}$, etc.

For $c$ and its preimages the above ambiguity can be dealt with by considering $c$ as a point of $\Delta$ or as a point of $\Delta^{\prime}$ and assigning different itineraries respectively. That is, if we take a preimage of $c$ then until it maps onto $c$ its itinerary is well-defined but at the time when it maps onto $c$ we either consider it as a point of $\Delta$ and assign 1 to it, or consider it as a point of $\Delta^{\prime}$ and assign 0 to it. For the forthcoming images of $c$ we again have no ambiguity ( 1 is assigned to all of them). In other words, to each preimage of $c$ exactly two itineraries are assigned as explained above. This implies that two $\Delta^{\prime}$-pullbacks with itineraries $\bar{i}=i_{0} i_{1} \ldots$ and $\bar{j}=j_{0} j_{1} \ldots$ have a point in common if and only if there exists $k$ such that $i_{0}=j_{0}, \ldots, i_{k-1}=j_{k-1}, i_{k}=1, j_{k}=0$ while $i_{r}=j_{r}=1$ for any $r>k$ (in particular, no more than two $\Delta^{\prime}$-pullbacks can intersect at one point). In what follows we denote the $\Delta^{\prime}$-pullback with itinerary $\bar{i}$ as $\Delta_{\bar{i}}$. Observe that since $\Delta$ and $\Delta^{\prime}$ have only one point - namely 
$c$ - in common, then any two $\Delta^{\prime}$-pullbacks (or $\Delta$ ) may have at most one point in common and no three $\Delta^{\prime}$-pullbacks (or $\Delta$ ) intersect (any common point of two $\Delta^{\prime}$-pullbacks maps onto $c$ while the two pullbacks in question map onto $\Delta$ and $\Delta^{\prime}$ ).

So far we have considered as examples points of $\Delta^{\prime}$-pullbacks (or $\Delta^{\prime}$-pullbacks themselves). To deal with other points of $J$ we need well-known facts about Julia sets of polynomials $P \in \mathcal{S}_{l c}$ (see, e.g., GMO99]); the notation introduced here will be used from now on. Let $p$ be the fixed point of $P$ belonging to $\Delta, \sigma$ be the angle doubling map on $S^{1},\left(\gamma, \gamma^{\prime}\right)$ (resp. $\left.\left[\gamma, \gamma^{\prime}\right]\right)$ be the open (resp. closed) counterclockwise circle arc from $\gamma$ to $\gamma^{\prime}$, and let $\overline{\gamma \gamma^{\prime}}$ be the chord connecting $\gamma$ and $\gamma^{\prime}$ in the disk. If $P^{\prime}(p)=e^{2 \pi i \rho}$ ( $\rho$ is irrational) then there exists a special rotational $\sigma$-invariant Cantor set $F \subset S^{1}$ such that $\left.\sigma\right|_{F}$ is no more than 2-to-1 semiconjugate to the irrational rotation by the angle $2 \pi \rho$ BS94. More precisely, call an arc complementary to $F$ an $F$-hole. Then the semiconjugacy maps the endpoints of every $F$-hole into one point and otherwise is one-to-one. The most important $F$-hole is the longest one which is the half-circle $(\beta, \alpha)$ with the endpoints denoted below by $0<\alpha<1 / 2$ and $\beta=\alpha+1 / 2$; the chord $\overline{\alpha \beta}$ connecting $\alpha$ and $\beta$ is called the critical leaf (diameter). Other $F$-holes are preimages of $(\beta, \alpha)$.

The limit set $F=\omega(\alpha)$ is exactly the set of angles whose entire orbits are contained in $[\alpha, \beta]$; also, the angles in $F$ are exactly the angles whose rays land at points of $S=\partial \Delta$. The endpoints of an $F$ hole can be denoted by $\alpha_{-n}, \beta_{-n}$ (since they are appropriate preimages of $\alpha$ and $\beta$ ). Both rays $R_{\alpha_{-n}}$ and $R_{\beta_{-n}}$ land at the point $c_{-n}$, the $n$-th pullback of $c$ belonging to $\Delta$. We need the following simple fact.

Lemma 2.1. Suppose that two angles $\alpha^{\prime \prime} \neq \beta^{\prime \prime}$ are given none of which maps into $F$ by any power of $\sigma$. Then there exists $m$ such that $\sigma^{m}\left(\alpha^{\prime \prime}\right)$ and $\sigma^{m}\left(\beta^{\prime \prime}\right)$ are separated by the critical leaf $\overline{\alpha \beta}$.

Proof. Define $d\left(\theta, \theta^{\prime}\right)$ as the length of the shortest arc between $\theta$ and $\theta^{\prime}$ (we normalize the circle so that its length is equal to 1 ). It is easy to see that $d\left(\sigma(\theta), \sigma\left(\theta^{\prime}\right)\right)=T\left(d\left(\theta, \theta^{\prime}\right)\right)$ where $T:[0,1 / 2] \rightarrow[0,1 / 2]$ is the appropriate scaling of the full tent map. Since $T(x)>x$ for $0<x<1 / 3$, there exists $m$ such that $d\left(\sigma^{m}\left(\alpha^{\prime \prime}\right), \sigma^{m}\left(\beta^{\prime \prime}\right)\right) \geq 1 / 3$. If $d\left(\sigma^{m}\left(\alpha^{\prime \prime}\right), \sigma^{m}\left(\beta^{\prime \prime}\right)\right)=1 / 2$ then since $\alpha^{\prime \prime}, \beta^{\prime \prime}$ are not preimages of $\sigma(\alpha)$ we see that $\sigma^{m}\left(\alpha^{\prime \prime}\right), \sigma^{m}\left(\beta^{\prime \prime}\right)$ are separated by $\overline{\alpha \beta}$ and we are done. Assume that $\sigma^{m}\left(\alpha^{\prime \prime}\right), \sigma^{m}\left(\beta^{\prime \prime}\right)$ are not separated by $\overline{\alpha \beta}$ and $d\left(\sigma^{m}\left(\alpha^{\prime \prime}\right), \sigma^{m}\left(\beta^{\prime \prime}\right)\right)<$ $1 / 2$. Since the longest complementary arcs to the set $\sigma^{-1}(F)$ are of length $1 / 4$, we see that $\sigma^{m}\left(\alpha^{\prime \prime}\right), \sigma^{m}\left(\beta^{\prime \prime}\right)$ belong to two distinct complementary arcs of $F \cup F+1 / 2$ located on one side of $\overline{\alpha \beta}$. Thus after 
several steps the $\sigma$-image of, say, $\alpha^{\prime \prime}$ will belong to $(\beta, \alpha)$ while the corresponding image of $\beta^{\prime \prime}$ will still be inside $(\alpha, \beta)$. This means that these two images of $\alpha^{\prime \prime}, \beta^{\prime \prime}$ will be separated by $\overline{\alpha \beta}$.

Call a point $y \in J$ a local cutpoint of $J$ if the point $y$ is a cutpoint of some connected neighborhood $U$ of $y$ in $J$. The union of boundaries of $\Delta$ and all $\Delta^{\prime}$-pullbacks forms the set of all local cutpoints of $J$. The remaining points of $J$ are called the endpoints of $J$, or, more informally, the "dust". We say that a connected set $X$ connects a connected set $A$ and a connected set $B$ if the union $A \cup B \cup X$ is connected (in practice we use this term when $A$ and $B$ are disjoint). Also, by a string of $\Delta^{\prime}$-pullbacks we mean a countable collection of $\Delta^{\prime}$-pullbacks concatenated to each other (so that consecutive pullbacks in the string intersect over exactly one point). Lemma 2.2 studies how dust points in $J$ are connected to $\Delta$.

Lemma 2.2. Let $y \in J$ be a dust point with itinerary $\bar{i}=\left(i_{0} i_{1} \ldots\right)$. Then there exists a unique string $S_{y}$ of $\Delta^{\prime}$-pullbacks which connects $\Delta$ and $y$. Denote the $\Delta^{\prime}$-pullbacks in $S_{y}$ as follows: $\Delta^{1}(y)$ is the closest to $\Delta$ (in the sense of the spatial order on the string), $\Delta^{2}(y)$ is the second one, etc. Then the itinerary of $\Delta^{j}(y)$ is obtained from the itinerary $\bar{i}=i_{0} i_{1} \ldots$ of $y$ as follows: choose the $j$-th appearance of 0 in $\bar{i}$, keep all the entries before that, and replace all other entries in $\bar{i}$ by 1 .

Proof. Since $J$ is locally connected, it is arcwise connected. Hence there exists an arc (homeomorphic image of the interval $[0,1]) I^{\prime} \subset J$ connecting $y$ and $c$. Let us show that $I^{\prime} \cap \Delta=I^{\prime} \cap S$ is connected (recall, that $S=\partial \Delta$ ). Indeed, suppose that there exists an arc in $J$ whose endpoints belong to $S$ while otherwise the arc is disjoint from $S$. Then it follows that parts of $S$ are "shielded" from infinity by this arc, i.e. are not accessible from infinity, a contradiction. Hence such arcs in $J$ do not exist which shows that $I^{\prime} \cap S$ is either an arc or the point $c$. If $I^{\prime} \cap S=\{c\}$ is a point set $\pi(y)=c$, otherwise let $\pi(y)$ be the other endpoint of $I^{\prime} \cap S$. Observe that by the above argument such point is unique so that $\pi$ is well-defined. Denote the arc connecting $y$ and $\pi(y)$ by $I$; then $I \cap S=\pi(y)$. Intuitively, one can think of the point $\pi(y)$ as a "projection" of $y$ into $S$.

Since all points of $I$ except for $y$ are not from the dust, it follows that $I \backslash\{y, \pi(y)\}$ is contained in the union of some $\Delta^{\prime}$-pullbacks. The union of these $\Delta^{\prime}$-pullbacks forms the desired string $S_{y}$. Observe, that the string $S_{y}$ is unique by the same geometric argument as above - otherwise some points of the Julia set are not accessible from infinity because they will be "shielded" from infinity by other connected subsets of $J$ (these 
subsets will be parts of the two strings which hypothetically connect $\Delta$ and $y$ and will form the boundary of a simply connected domain in the plane containing points of $J$ ). A similar geometric argument is often used in the paper, so we explain it here in detail while simply alluding to it in the future.

By the definition we do not include $\Delta$ in $S_{y}$ and begin $S_{y}$ from $\Delta^{1}(y)$, the $\Delta^{\prime}$-pullback closest to $\Delta$ in $S_{y}$. Thus, $\Delta^{1}(y)$ is either $\Delta^{\prime}$ or a pullback of $\Delta^{\prime}$ attached to $\Delta$. Therefore $\Delta^{1}(y)$ has itinerary $1_{k_{1}} 01_{\infty}$ with $k_{1} \geq 0$ digits 1 to begin with (if $k_{1}=0$ then $\Delta^{1}(y)=\Delta^{\prime}$ ). The next pullback $\Delta^{2}(y)$ in the string $S_{y}$ is attached to $\Delta^{1}(y)$, hence its itinerary coincides with that of $\Delta^{1}(y)$ until $\Delta^{1}(y)$ maps onto $\Delta$. At this moment $\Delta^{2}(y)$ becomes the closest to $\Delta$ pullback of $\Delta^{\prime}$ in the appropriate image of $S_{y}$ and the process repeats itself with the only difference that now the image of $\Delta^{2}(y)$ needs $k_{2}$ steps to move around $\Delta$ until it finally gets mapped onto $\Delta^{\prime}$ and then onto $\Delta$. This argument yields that the "spatially" $j$-th pullback $\Delta^{j}(y)$ of $\Delta^{\prime}$ in $S_{y}$ has itinerary $1_{k_{1}} 01_{k_{2}} 0 \ldots 1_{k_{j}} 01_{\infty}$. It has to coincide with the itinerary of $y$ until the first time $\Delta^{j}(y)$ maps onto $\Delta$. Also, numbers $k_{i}$ may be equal to zero. Hence the itinerary of $\Delta^{j}(y)$ is obtained from the itinerary $\bar{i}=i_{0} i_{1} \ldots$ of $y$ as follows: choose the $j$-th appearance of 0 in $\bar{i}$, keep all the entries before that, and replace all other entries in $\bar{i}$ by 1 .

As an example consider the periodic point $x$ with itinerary $\bar{i}=$ $\{011\}_{\infty}$. Then the following are the pullbacks of $\Delta^{\prime}$ forming the string $S_{x}: \Delta^{1}(x)=\Delta_{01_{\infty}}, \Delta^{2}(x)=\Delta_{01101_{\infty}}, \Delta^{3}(x)=\Delta_{01101101_{\infty}}$ etc. By Lemma 2.1 any two distinct periodic points $u \in J$ and $v \in J$ have distinct itineraries (it is enough to consider rays landing at $u$ and $v$ ). Therefore their strings $S_{u}$ and $S_{v}$ may have a certain initial piece in common (perhaps empty), and then will separate. For example, let $z$ be the periodic point of itinerary $\{0110111\}_{\infty}$. Then the string $S_{z}$ consists of the following pullbacks of $\Delta^{\prime}: \Delta^{1}(z)=\Delta_{01_{\infty}}, \Delta^{2}(z)=$ $\Delta_{01101_{\infty}}, \Delta^{3}(z)=\Delta_{011011101_{\infty}}, \ldots$ Comparing $S_{x}$ and $S_{z}$ we see that $\Delta^{1}(x)=\Delta^{1}(z), \Delta^{2}(x)=\Delta^{2}(z)$, but $\Delta^{3}(x) \neq \Delta^{3}(z)$. It follows that $\Delta^{3}(x)$ and $\Delta^{3}(z)$ are disjoint (both are attached to the same $\Delta^{\prime}$-pullback but at distinct points, hence if they meet then some points of the Julia set will not be accessible from infinity). From this moment on the strings $S_{x}$ and $S_{z}$ go their own ways, converging to $x$ and $z$ respectively.

Consider now the dynamics of the string $S_{x}$. Since $x$ is of period 3 then $S_{x}$ must cover itself under $P^{3}$ while $P^{3}\left(S_{x}\right)$ contains $\Delta$. In fact, already the first application of $P$ restricted onto $S_{x}$ maps $\Delta^{1}(x)$ onto $\Delta$. Then $S_{x}$ rotates about $\Delta$ for one step and on the next step $S_{x}$ maps over itself. Thus, $P^{3}\left(\Delta^{2}(x)\right)=\Delta^{1}(x), P^{3}\left(\Delta^{3}(x)\right)=\Delta^{2}(x)$ etc. 
In other words, $P^{3}$ shifts the pullbacks in $S_{x} 1$ pullback "down" (i.e. closer to $\Delta$ ). The number 1 is then called the basic length of $S_{x}$; the string $S_{x}$ consists of countably many fragments whose length is 1 and who are shifted by $P^{3}$ one onto another closer to $\Delta$ except for the first fragment of $S_{x}$ which maps by $P^{3}$ onto $\Delta$.

In the case of the string $S_{z}$ the picture is a bit more complicated, however it has essentially the same properties (in our description of the dynamics of $S_{z}$ we skip discussing simple rotations of $S_{z}$ around $\Delta)$. It is easy to check that first $P^{3}$ shifts $\Delta^{2}(z)$ onto $\Delta^{1}(z)$ while $\Delta^{3}(z)=\Delta_{011011101_{\infty}}$ maps onto $\Delta_{011101_{\infty}}$. Then $P^{4}$ maps $\Delta_{011101_{\infty}}$ onto $\Delta^{\prime}=\Delta^{1}(z)$ and the entire string $S_{z}$ finally covers itself. In other words, since the periodic fragment of the itinerary of $z$ contains two zeros, then $P^{7}$ shifts the pullbacks in $S^{z}$ down (closer to $\Delta$ ) by 2 pullbacks. In this case the basic length of $S_{z}$ is 2 ; the string $S_{z}$ consists of countably many fragments whose length is 2 and who are shifted by $P^{7}$ one onto another closer to $\Delta$ except for the first fragment of $S_{z}$ which maps by $P^{7}$ onto $\Delta$. Clearly, $P^{7}$ maps the first fragment of $S_{z}$ onto $\Delta$ as a continuous map (there have to be critical points), but otherwise the map $P^{7}$ shifts fragments in $S_{z}$ homeomorphically.

In general, given a periodic orbit $y$ of period $k$ whose itinerary has the minimal periodic fragment with $l$ zeros we see that the map $P^{k}$ shifts the $\Delta^{\prime}$-pullbacks in $S_{y}$ down (closer to $\Delta$ ) by $l$ pullbacks. In this case the first fragment of $S_{y}$ is $\Delta^{1}(y) \cup \cdots \cup \Delta^{l}(y)$, the second fragment of $S_{y}$ is the union $\Delta^{l+1}(y) \cup \cdots \cup \Delta^{2 l}(y)$, etc. Observe that all the fragments in $S_{y}$ are in fact the pullbacks of the first fragment by the appropriate branch of the inverse function to $P^{l}$, and from the second fragment on all the fragments in $S_{y}$ are disjoint from $\Delta$. Moreover, since in this case the critical limit set $\omega_{P}(c)$ coincides with $\Delta$, we see that by well-known shrinking properties of pullbacks under polynomial/rational maps (see, e.g., Shrinking Lemma [LM97]) the diameters of the pullbacks in $S_{y}$ converge to 0 . If diameters of the sets in a sequence converge to 0 then the sets are said to form a null sequence; it follows that in this case the fragments in $S_{y}$ described above form a null sequence. Of course, in the case at hand any string $S_{\zeta}$ converges to the point $\zeta$ defining it - after all, the Julia set $J$ is locally connected. However even in the case of basic Cremer Julia sets (which are not locally connected) these ideas, with some modifications, still apply.

Let us go back to the example we have already partially considered before, i.e. to the periodic points $x, z$ and their strings $S_{x}, S_{z}$. As we have seen, $S_{x}$ and $S_{z}$ converge to the periodic points $x$ and $z$ respectively. On the other hand, we have seen that the strings $S_{x}$ and $S_{z}$ 


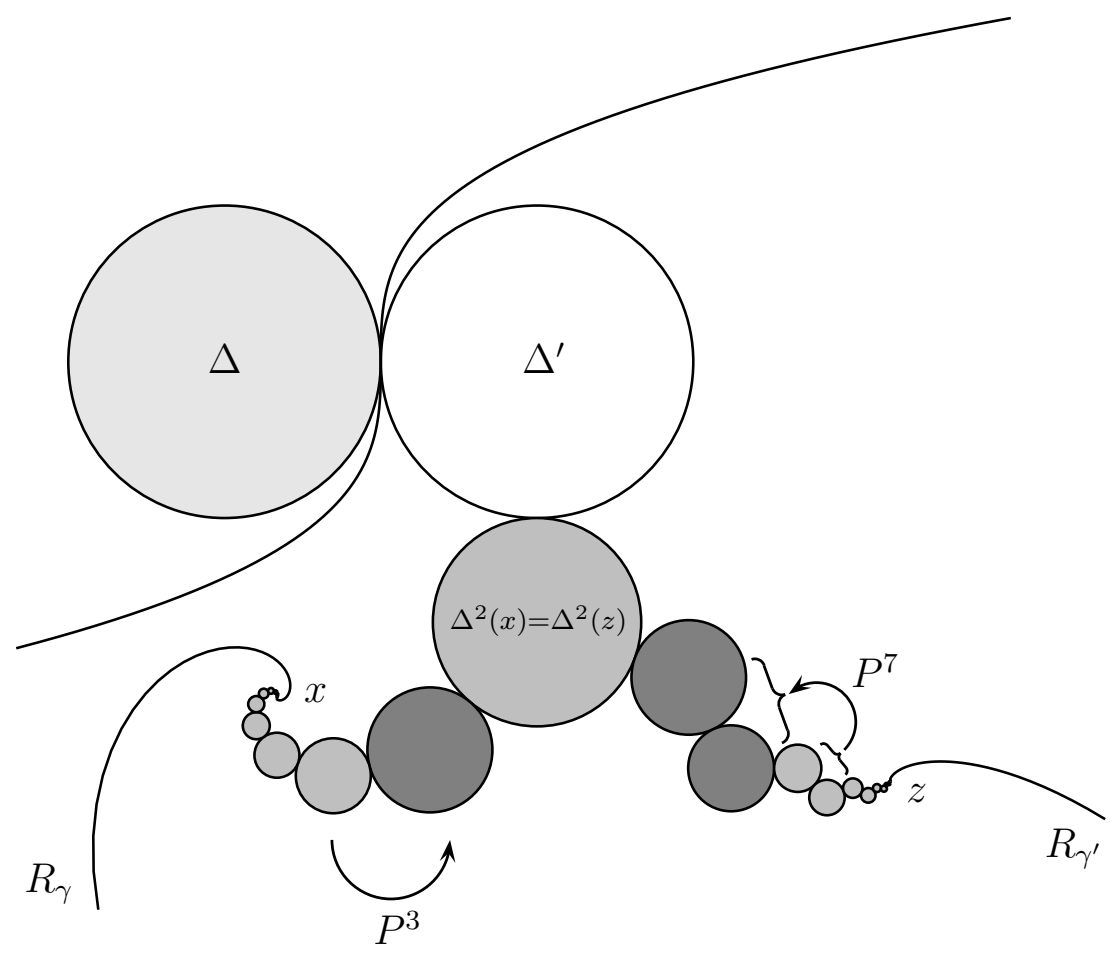

FiguRE 3. Illustration, for the golden mean basic Siegel Julia set, of the examples of periodic points $x$ and $z$ considered in the text, together with the associated strings of $\Delta$-pullbacks.

separate after the pullback $\Delta^{2}(x)=\Delta^{2}(z)=\Delta_{01101_{\infty}}$. Let $R_{\gamma}, R_{\gamma^{\prime}}$ be the external rays landing at $x$ and $z$ respectively. Denote by $I$ the open arc between $\gamma$ and $\gamma^{\prime}$ contained in the 0 -semicircle. Denote by $\hat{S}_{x}$ the closure of the "tail" of $S_{x}$ taken from $\Delta_{01101 \infty}$ to the point $x$ and by $\hat{S}_{z}$ the closure of the "tail" of $S_{z}$ taken from $\Delta_{01101 \infty}$ to the point $z$ (so that $\left.\hat{S}_{x} \cap \hat{S}_{z}=\Delta_{01101 \infty}\right)$. Then the union of $\hat{S}_{x}, \hat{S}_{z}, R_{\gamma}$ and $R_{\gamma}^{\prime}$ is a "fork" which encloses a wedge containing all rays $R_{\theta}, \theta \in I$. The impression of $\theta \in I$ must be contained in the closure of this wedge and therefore is disjoint from $\Delta$. Again, for the polynomials in $\mathcal{S}_{l c}$ this fact can be shown in a much easier way. However the argument is valuable because it applies in more general situations.

Let $\sigma_{2}$ be the one-sided shift on the space of all sequences with symbols 1 and 0 , and let $A_{n}=\sigma_{2}^{-n}\left(1_{\infty}\right)$. Notice that $A_{n}$ includes all $\sigma_{2}$-preimages of $1_{\infty}$ of order less than or equal to $n$. Consider elements 
of $A_{n}$ as vertices of a graph connected by edges if and only if the $\Delta^{\prime}$ pullbacks with these itineraries intersect. Clearly, this makes $A_{n}$ into a tree (if there is a loop in $A_{n}$ then as before there must be points of $J$ shielded from the infinity, a conradiction). From now on we always consider $A_{n}$ endowed with the tree structure. Suppose that for each $\bar{s} \in A_{n}$ a set $M_{\bar{s}}$ is given and $g: \cup M_{\bar{t}} \rightarrow \cup M_{\bar{t}}$ is a function such that 1) $g\left(M_{\bar{s}}\right) \subset M_{\sigma_{2}(\bar{s})}$, and 2) $M_{\bar{s}} \cap M_{\bar{t}} \neq \emptyset$ if and only if $\Delta_{\bar{s}} \cap \Delta_{\bar{t}} \neq \emptyset$. Then we say that $\left(g,\left\{M_{\bar{s}}, \bar{s} \in A_{n}\right\}\right)$ has the same (dynamical) intersection pattern as $\left\{\Delta_{\bar{s}}, \bar{s} \in A_{n}\right\}$. It follows from the results of [BC06] (see below) that for certain basic Cremer polynomials $f$ there is an invariant continuum $M$ which plays the role of the Siegel disk $\Delta$ above in the sense that the intersection pattern of $M$ and all appropriately defined $M^{\prime}$-pullbacks of order $n$ and the intersection pattern of $\Delta$ and all $\Delta^{\prime}$ pullbacks of order $n$ are the same.

2.3. Basic Cremer polynomials. Let us introduce terminology and notation. Let $P_{\alpha}(z)=e^{2 \pi i \alpha} z+z^{2}$. Clearly, the critical point of $P_{\alpha}$ is $c_{\alpha}=(-.5) e^{2 \pi i \alpha}$; set $M_{\alpha}=\omega\left(c_{\alpha}\right)$ and call it the critical limit set of $P_{\alpha}$. It is well-known (see, e.g., Mn93]) that if $P_{\alpha}$ is a basic Cremer polynomial then its Cremer fixed point $p_{\alpha}$ belongs to $M_{\alpha}$. The next lemma is obtained in [C05].

Lemma 2.3 (Childers). If $P_{\alpha}$ is a basic Cremer polynomial then the set $M_{\alpha}$ is a continuum.

The construction in the next section aims at proving that certain Julia sets of positive area are solar. We need the following definition: an angle $\theta$ is said to be of bounded type if there exists $K$ such that in the continued fraction expansion $\left[a_{1}, a_{2}, \ldots\right]$ of $\theta$ we have $a_{i}<K$ for all $i$. Consider the polynomial $P_{\theta}(z)$ assuming that $\theta$ is of bounded type. Then $P_{\theta}$ has an invariant closed Siegel disk $\Delta_{\theta}, \partial \Delta_{\theta}=M_{\theta}$ is a simple closed curve and $J_{P_{\theta}}$ is locally connected [Pet96]. For every $N$, define $\tilde{S}_{N}$ as the set of all $\theta$ 's with $a_{i} \geq N, i=1,2, \ldots$. Given two sets $A, B$ let us denote by $\partial[A, B]$ the number $\sup \{\operatorname{dist}(a, B): a \in A\}$ (thus, if $\partial[A, B]=\varepsilon$ then the set $A$ is contained in the closed $\varepsilon$-neighborhood of $B$ ). Theorem 2.4 is proven in [BC06]. The key tool in the proof is the use of Inou-Shishikura's results on near parabolic renormalization [IS].

Theorem 2.4 (Buff-Chéritat). There exists $N$ such that for every $\theta \in$ $\tilde{S}_{N}$ of bounded type and every $\varepsilon>0$ if $\alpha \in \tilde{S}_{N}$ is sufficiently close to $\theta$ then $\partial\left[M_{\alpha}, \Delta_{\theta}\right]<\varepsilon$ and $M_{\alpha} \neq J_{P_{\alpha}}$. Moreover, there exist basic Cremer polynomials $P_{\alpha}$ with $\alpha \in \tilde{S}_{N}$ arbitrarily close to $\theta$ and with positive area Julia sets. 
In what follows we will use the notation introduced above.

\section{MAIN THEOREM}

The aim of this section is to prove Theorem 1.2. From now on we fix $N$ as in Theorem [2.4. For a polynomial $P_{\alpha}$, we denote by $\psi_{\alpha}$ the involution which maps any $z$ to the other preimage of $P_{\alpha}(z)$. Set $\psi_{\alpha}\left(M_{\alpha}\right)=M_{\alpha}^{\prime}$. The following lemma uses self-explanatory notation.

Lemma 3.1. Given $n, \theta \in \tilde{S}_{N}$ of bounded type, and $\varepsilon>0$ there exists a neighborhood $U$ of $\theta$ such that for any $\alpha \in \tilde{S}_{N} \cap U$ we have $P_{\alpha}^{i}\left(c_{\alpha}\right) \notin M_{\alpha}^{\prime}, i \leq n$, and there exists a collection of well-defined $M_{\alpha}^{\prime}$ pullbacks $M_{\alpha, \bar{j}}, \bar{j} \in A_{n}$ by $P_{\alpha}$ such that $\partial\left[M_{\alpha, \bar{j}}, \Delta_{\theta, \bar{j}}\right]<\varepsilon$ for any $\bar{j} \in A_{n}$ and the $M_{\alpha}^{\prime}$-pullbacks $\left\{M_{\alpha, \bar{j}}, \bar{j} \in A_{n}\right\}$ have the same intersection pattern as $\Delta_{\theta}^{\prime}$-pullbacks $\left\{\Delta_{\theta, \bar{j}}, \bar{j} \in A_{n}\right\}$.

Proof. The proof is based upon Theorem 2.4 and continuity arguments. By Theorem 2.4, $M_{\alpha}^{\prime}$ is very close to $\Delta_{\theta}^{\prime}$ for $\alpha \in \tilde{S}_{N}$ sufficiently close to $\theta$. Since $P_{\theta}^{i}\left(c_{\theta}\right) \notin \Delta_{\theta}^{\prime}$, it follows then that $P_{\alpha}^{i}\left(c_{\alpha}\right) \notin M_{\alpha}^{\prime}, i \leq n$ for $\alpha \in \tilde{S}_{N}$ sufficiently close to $\theta$ (so that an $i$-th pullback of $M_{\alpha}^{\prime}$ is mapped 1-to-1 if $i \leq n$ ). Moreover, by continuity and by Theorem 2.4 given an itinerary $\bar{j} \in A_{n}$ we can correctly define $M_{\alpha}^{\prime}$-pullbacks $M_{\alpha, \bar{j}}$ by $P_{\alpha}$ corresponding to $\Delta_{\theta}^{\prime}$-pullbacks $\Delta_{\theta, \bar{j}}$ by $P_{\theta}$ and guarantee that 1) $\partial\left[M_{\alpha, \bar{j}}, \Delta_{\theta, \bar{j}}\right]<\varepsilon$ for any $\bar{j} \in A_{n}$, and hence 2) for two itineraries $\bar{s} \in A_{n}, \bar{t} \in A_{n}$ if $\Delta_{\theta, \bar{s}} \cap \Delta_{\theta, \bar{t}}=\emptyset$ then $M_{\alpha, \bar{s}} \cap M_{\alpha, \bar{t}}=\emptyset$. Let us show that if $\Delta_{\theta, \bar{s}} \cap \Delta_{\theta, \bar{t}} \neq \emptyset$ then $M_{\alpha, \bar{s}} \cap M_{\alpha, \bar{t}} \neq \emptyset$. Observe that since $A_{n}$ is a tree then if we remove the edge in $A_{n}$ connecting $\bar{s}$ and $\bar{t}$ then $A_{n}$ falls into two trees, $A_{\bar{s}}$ and $A_{\bar{t}}$. Now, it is easy to see that the union of all pullbacks $M_{\alpha, \bar{r}}, \bar{r} \in A_{n}$ is the set $P_{\alpha}^{-n}\left(M_{\alpha}\right)$ which is connected because the entire orbit of $c_{\alpha}$ is contained in $M_{\alpha}\left(c_{\alpha}\right.$ is recurrent for $P_{\alpha}$ and $\left.M_{\alpha}=\omega\left(c_{\alpha}\right)\right)$. On the other hand suppose that $M_{\alpha, \bar{s}} \cap M_{\alpha, \bar{t}}=\emptyset$. Then the unions $M_{\alpha, A_{\bar{s}}}=\cup_{\bar{i} \in A_{\bar{s}}} M_{\alpha, \bar{i}}$ and $M_{\alpha, A_{\bar{t}}}=\cup_{\bar{i} \in A_{\bar{t}}} M_{\alpha, \bar{i}}$ are disjoint because they could only intersect over $M_{\alpha, \bar{s}} \cap M_{\alpha, \bar{t}}$ which is empty. This contradiction shows that $M_{\alpha, \bar{s}} \cap M_{\alpha, \bar{t}} \neq \emptyset$ as desired.

We are now ready to prove Theorem 1.2 . However it will be convenient to first prove a simple technical lemma.

Lemma 3.2. Let $P$ be a basic Cremer polynomial and $K \subset J$ be a continuum such that for some $k>0$ we have that $P^{-k}(K) \cap K \neq \emptyset$ and $P^{i}(c) \notin P^{k}(K)$ for any $i \geq 0$. Then there exists a unique sequence $K(0)=K, K(-1), \ldots$ of $K$ pullbacks by $P^{k}$ such that $K(-i) \cap K(-i-$ $1) \neq \emptyset$ and $P^{k}(K(-i-1))=K(-i)$ for all $i \geq 0$. Moreover, if $P^{2 k}(K)$ 
is disjoint from $K$ then in the sequence of sets $P^{2 k}(K), P^{k}(K), K, \ldots$ non-empty intersections are only possible between two consecutive sets.

Proof. Since $J$ is a non-separating one-dimensional continuum, $J$ is a tree-like continuum (i.e., for each $\varepsilon>0$ there exists a map $\phi: J \rightarrow T$ where $T$ is a finite tree such that for each $t \in T$ the diameter of $\left.\phi^{-1}(t)<\varepsilon\right)$. Since $P^{i}(c) \notin K$ then it is clear that for every $i$ the family of $K$-pullbacks by $P^{k}$ consists of $2^{k i}$ pairwise disjoint continua contained in $J$. Since $P^{-k}(K) \cap K \neq 0$, there exists a $K$-pullback $K(-1)$ of order 1 by $P^{k}$ non-disjoint from $K$. Such pullback is unique. Indeed, otherwise there exists another $K$-pullback $K^{\prime}$ of order 1 by $P^{k}$ which is not disjoint from $K$. Set $K(-1) \cup K \cup K^{\prime}=Y$. Then $\left.P^{k}\right|_{Y}$ is not 1-to-1 which implies by [H96] that $\left.P^{k}\right|_{Y}$ has a critical point $y \in Y$. This means that for some $j, 0 \leq j \leq k-1$ we have $P^{j}(y)=c$. If $y \in K(-1) \cup K^{\prime}$ then this implies that $P^{2 k-j}(c) \in P^{k}(K)$, a contradiction to the assumptions. On the other hand, if $y \in K$ then $P^{k-j}(c) \in P^{k}(K)$, again a contradiction to the assumptions. Hence such point $y \in Y$ does not exist, and $K(-1)$ is unique. Since $K(-1)$ clearly satisfies the same assumptions as $K$ itself we see that the desired sequence of pullbacks of $K$ exists and is unique.

Suppose that $P^{2 k}(K) \cap K=\emptyset$. Then $P^{k}(K) \cap K(-1)=\emptyset$ and for any $i \geq 0$ we have $K(-i) \cap K(-i-2)=\emptyset$ (otherwise we apply the appropriate power of $P^{k}$ to get a contradiction). Let us show that then $K(-j) \cap P^{2 k}(K)=\emptyset$ for any $j \geq 0$. Indeed, otherwise choose the minimal such $j$ that $K(-j) \cap P^{2 k}(K) \neq \emptyset$. Then $j \geq$ 1 and by the choice of $j$ the only non-empty intersections among sets $P^{2 k}(K), P^{k}(K), K, \ldots, K(-j)$ are intersections among consecutive pullbacks and the intersection $K(-j) \cap P^{2 k}(K) \neq \emptyset$. Set $P^{2 k}(K) \cup$ $P^{k}(K) \cup \cdots \cup K(-j+1)=E$. Then

$$
E \cap K(-j)=\left[P^{2 k}(K) \cap K(-j)\right] \cup[K(-j+1) \cap K(-j)]
$$

is disconnected as the union of two disjoint non-empty continua. However all continua are contained in a non-separating continuum $J$ with empty interior. Hence the intersection of any two sub-continua of $J$ must be connected. It follows that $K(-j) \cap P^{k}(K)=\emptyset$ for any $j \geq 1$ as desired.

To prove Theorem 1.2 we need the following construction. Choose two $P_{\theta}$-periodic points $u$ and $v$ of periods $k$ and $l$ respectively. Then depending on their itineraries the strings $S_{u}, S_{v}$ of $\Delta_{\theta}^{\prime}$-pullbacks will have a few common pullbacks and then, starting at the last common pullback, will consist of two pairwise disjoint sequences of pullbacks. 
Clearly, the points $u$ and $v$ can be chosen so that the strings $S_{u}$ and $S_{v}$ have at least two common $\Delta_{\theta}^{\prime}$-pullbacks. For example, if $u=x$ has itinerary $\{011\}_{\infty}$ and $v=z$ has itinerary $\{0110111\}_{\infty}$ then the strings $S_{x}$ and $S_{z}$ have two pullbacks $\Delta_{\theta}^{\prime}=\Delta_{\theta, 01_{\infty}}$ and $\Delta_{\theta, 01101_{\infty}}$ in common, yet from the third pullback on the strings $S_{x}$ and $S_{z}$ are disjoint. In any case, and this is important for what follows, the last common pullback of the strings $S_{x}$ and $S_{z}$ is $\Delta_{\theta, 01101_{\infty}}$, and it is disjoint from $\Delta_{\theta}$.

Let us assume that the basic length of $S_{u}$ is $w$, the basic length of $S_{v}$ is $q$, and the initial finite string $F$, common to both $S_{u}$ and $S_{v}$, consists of $m \geq 2 \Delta_{\theta}^{\prime}$-pullbacks. For simplicity and without loss of generality assume that $m<\min (w, q)$. Denote the last common $\Delta_{\theta}^{\prime}$-pullback in $F$ by $L$. Consider $w \Delta_{\theta}^{\prime}$-pullbacks in $S_{u}$ immediately following $F$ and denote their union by $\hat{F}_{u}$; also, denote the union of $w$ $\Delta_{\theta}^{\prime}$-pullbacks in $S_{u}$ immediately following $\hat{F}_{u}$ by $F_{u}$. Similarly, consider $q \Delta_{\theta}^{\prime}$-pullbacks in $S_{v}$ immediately following $F$ and denote their union by $\hat{F}_{v}$; also, denote the union of $q \Delta_{\theta}^{\prime}$-pullbacks in $S_{v}$ immediately following $\hat{F}_{v}$ by $F_{v}$. By Lemma 3.2 there exists a string of $F_{u}$-pullbacks by $P_{\theta}^{k} F_{u}(0)=F_{u}, F_{u}(-1), \ldots$ such that $S_{u}=F \cup \hat{F}_{u} \cup\left(\cup_{i=0}^{\infty} F_{u}(-i)\right)$ and a string of $F_{v}$-pullbacks by $P_{\theta}^{l} F_{v}(0)=F_{v}, F_{v}(-1), \ldots$ such that $S_{v}=F \cup \hat{F}_{v} \cup\left(\cup_{i=0}^{\infty} F_{v}(-i)\right)$.

Set $n=m+3 k+3 l$. We choose $\alpha \in \tilde{S}_{N}$ very close to $\theta$ so that a few conditions are satisfied. By Theorem 2.4 we may assume that the area of $J\left(P_{\alpha}\right)$ is positive and that $P_{\alpha}$ is a basic Cremer polynomial. We use Lemma 3.1 to guarantee that $\partial\left[M_{\alpha}, \Delta_{\theta}\right]$ is so small that the first $n$ iterates of $c_{\alpha}$ avoid $M_{\alpha}^{\prime}$ (so that $M_{\alpha}^{\prime}$-pullbacks by $P_{\alpha}$ of order at most $n$ map to $M_{\alpha}^{\prime}$ univalently), all $M_{\alpha}^{\prime}$-pullbacks by $P_{\alpha}$ of order $n$ are very close to the corresponding $\Delta_{\theta}^{\prime}$-pullbacks by $P_{\theta}$ and the intersection patterns of $\Delta_{\theta, \bar{i}}^{\prime}, \bar{i} \in A_{n}$ and $M_{\alpha, \bar{i}}^{\prime}, \bar{i} \in A_{n}$ are the same. Hence we may talk about the strings $F^{\alpha}, \hat{F}_{u}^{\alpha}, \hat{F}_{v}^{\alpha}, F_{u}^{\alpha}, F_{v}^{\alpha}$ of $M_{\alpha}^{\prime}$-pullbacks by $P_{\alpha}$ which correspond to the strings $F, \hat{F}_{u}, \hat{F}_{v}, F_{u}, F_{v}$, and all the sets $F^{\alpha}, \hat{F}_{u}^{\alpha}, \hat{F}_{v}^{\alpha}, F_{u}^{\alpha}, F_{v}^{\alpha}$ are continua. Denote by $L^{\alpha}$ the $M_{\alpha}^{\prime}$-pullback corresponding to the $\Delta_{\theta^{-}}^{\prime}$ pullback $L$. Recall that since $m \geq 2$ then $L \cap \Delta_{\theta}=\emptyset$ and hence (by the choice of $\alpha)$ we may assume that $M_{\alpha} \cap\left(L^{\alpha} \cup \hat{F}_{u}^{\alpha} \cup \hat{F}_{v}^{\alpha}\right)=\emptyset$.

Consider the set $F_{u}^{\alpha}$. Then $P_{\alpha}^{k}\left(F_{u}^{\alpha}\right)=\hat{F}_{u}^{\alpha}$ is disjoint from $M_{\alpha}=\omega\left(c_{\alpha}\right)$ and hence $P_{\alpha}^{i}\left(c_{\alpha}\right) \notin P_{\alpha}^{k}\left(F_{u}^{\alpha}\right)$ for any $i \geq 0$. On the other hand, by the choice of $n$ we have that $P^{-k}\left(F_{u}^{\alpha}\right) \cap F_{u}^{\alpha} \neq \emptyset$. Hence by Lemma 3.2 there is a sequence of $F_{u}^{\alpha}$-pullbacks $F_{u}^{\alpha}=F_{u}^{\alpha}(0), F_{u}^{\alpha}(-1), \ldots$ by $P_{\alpha}^{k}$. Observe that $F_{u}^{\alpha}$ is disjoint from $P^{2 k}\left(F_{u}^{\alpha}\right)$ by the choice of $\alpha$ (clearly, $P^{2 k}\left(F_{u}^{\alpha}\right)=P_{\alpha}^{k}\left(\hat{F}_{u}^{\alpha}\right)$ is the string of $M_{\alpha}^{\prime}$-pullbacks by $P_{\alpha}^{k}$ connecting $L^{\alpha}$ 
and $M_{\alpha}$ united with $M_{\alpha}$ itself). By Lemma 3.2 we conclude that the set

$$
\hat{F}_{u}^{\alpha} \cup \bigcup_{i=0}^{\infty} F_{u}^{\alpha}(-i)=Q_{u}^{\alpha}
$$

is a chain of "concatenated" continua such that intersections among them are only possible between $\hat{F}_{u}^{\alpha}$ and $F_{u}^{\alpha}=F_{u}^{\alpha}(0)$ and between two consecutive pullbacks of $F_{u}^{\alpha}$.

Analogous claims can be proven for $\hat{F}_{v}^{\alpha}$ and $F_{v}^{\alpha}$. For the corresponding pullbacks of $F_{v}^{\alpha}$ we use similar notation and get the set

$$
\hat{F}_{v}^{\alpha} \cup \bigcup_{i=0}^{\infty} F_{v}^{\alpha}(-i)=Q_{v}^{\alpha},
$$

a chain of "concatenated" continua such that intersections among them are only possible between $\hat{F}_{v}^{\alpha}$ and $F_{v}^{\alpha}=F_{v}^{\alpha}(0)$ and between two consecutive pullbacks of $F_{v}^{\alpha}$.

It is easy to see that the sets $Q_{u}^{\alpha}$ and $Q_{v}^{\alpha}$ are disjoint. Indeed, suppose otherwise. Then we can choose minimal $r, s$ such that $F_{u}^{\alpha}(-r) \cap$ $F_{v}^{\alpha}(-s) \neq \emptyset$. It follows that $r>1$ and $s>1$. Then the continua $X=L^{\alpha} \cup\left(\cup_{i=0}^{r} F_{u}^{\alpha}(-i)\right)$ and $Y=L^{\alpha} \cup\left(\cup_{i=0}^{s} F_{v}^{\alpha}(-i)\right)$ have a disconnected intersection (it consists of $L^{\alpha}$ and a non-empty compact set $F_{u}^{\alpha}(-r) \cap F_{v}^{\alpha}(-l)$ disjoint from $\left.L^{\alpha}\right)$ despite the fact that they both are contained in the Julia set $J\left(P_{\alpha}\right)$ (recall that the Julia sets of basic Cremer polynomials are non-separating continua with empty interior).

Since $M_{\alpha} \cap\left(\hat{F}_{u}^{\alpha} \cup \hat{F}_{v}^{\alpha}\right)=\emptyset$ by the choice of $\alpha$ then by the Shrinking Lemma [LM97] we know that $\operatorname{diam}\left(F_{u}^{\alpha}(-i)\right) \rightarrow 0$ as $i \rightarrow \infty$ (i.e., $F_{u}^{\alpha}(-i), i=0,1, \ldots$ is a null-sequence). Hence by continuity any limit point $a$ of this sequence of sets is $P_{\alpha}^{k}$-fixed. Since there are finitely many $P_{\alpha}^{k}$-fixed points while the set of all limit points of the sequence of sets $F_{u}^{\alpha}(-i), i=0,1, \ldots$ is connected (recall, that this sequence of sets is a chain of "concatenated" continua) we conclude that the sequence $F_{u}^{\alpha}(-i), i=0,1, \ldots$ converges to a $P_{\alpha}^{k}$-fixed point which we will denote by $u^{\prime}$. Similarly, the sequence $F_{v}^{\alpha}(-i), i=0,1, \ldots$ converges to a $P_{\alpha}^{l}$ fixed point which we will denote by $v^{\prime}$.

Let us study possible intersections between some of these sets. Set $Z=P_{\alpha}^{k}\left(\hat{F}_{u}^{\alpha}\right)=P_{\alpha}^{l}\left(\hat{F}_{v}^{\alpha}\right)\left(Z\right.$ is the string of pullbacks of $M_{\alpha}^{\prime}$ connecting $L^{\alpha}$ and $M_{\alpha}$, united with $\left.M_{\alpha}\right)$. Lemma 3.2 implies that

$$
\left[Z \cup \hat{F}_{u}^{\alpha}\right] \cap Q_{u}^{\alpha}=\hat{F}_{u}^{\alpha}
$$

and 


$$
\left[Z \cup \hat{F}_{v}^{\alpha}\right] \cap Q_{v}^{\alpha}=\hat{F}_{v}^{\alpha}
$$

Let us show that $u^{\prime} \notin Z \cup Q_{u}^{\alpha}$. Indeed, since $u^{\prime}$ is a $P^{k}$-fixed point then $u^{\prime} \notin Q_{u}^{\alpha}$ because of the way sets $F_{u}^{\alpha}(-i), i \geq 0$ intersect. Suppose that $u^{\prime} \in Z$ and consider two continua, $X=\overline{Q_{u}^{\alpha}}$ and $Y=Z \cup \hat{F}_{u}^{\alpha}$. It follows that their intersection $X \cap Y=\left\{u^{\prime}\right\} \cup \hat{F}_{u}^{\alpha}$ is disconnected, a contradiction. Thus, $u^{\prime} \notin Z \cup Q_{u}^{\alpha}$. Similarly we can show that $v^{\prime} \notin Z \cup Q_{v}^{\alpha}$. Analogous arguments show that since the continua $\overline{Z \cup Q_{u}^{\alpha}}$ and $\overline{Z \cup Q_{v}^{\alpha}}$ must have a connected intersection then $u^{\prime} \notin Q_{v}^{\alpha}, v^{\prime} \notin Q_{v}^{\alpha}$

and $u^{\prime} \neq v^{\prime}$. Finally, since by the construction $M_{\alpha} \cap\left(L^{\alpha} \cup \hat{F}_{u}^{\alpha} \cup \hat{F}_{v}^{\alpha}\right)=\emptyset$ then $M_{\alpha} \cap \overline{L^{\alpha} \cup Q_{u}^{\alpha} \cup Q_{v}^{\alpha}}=\emptyset$ (notice that by the above $u^{\prime}, v^{\prime} \notin M_{\alpha}$.

Let $R_{\gamma}$ be the external ray for $P_{\alpha}$ landing at $u^{\prime}$ and $R_{\beta}$ be the external ray for $P_{\alpha}$ landing at $v^{\prime}$. The above implies that the union

$$
\overline{L_{\alpha} \cup Q_{u}^{\alpha} \cup Q_{v}^{\alpha}} \cup R_{\gamma} \cup R_{\beta}
$$

cuts the plane into two open half-planes $W$ and $H$, one of which (say, $W$ ) contains $M_{\alpha}$ (and therefore the Cremer point $p_{\alpha}$ of $P_{\alpha}$ ). Choose any external angle $\tau$ whose external ray $R_{\tau}$ is contained in $H$. Then it follows that the impression of $R_{\tau}$ is contained in $\overline{L_{\alpha} \cup Q_{u}^{\alpha} \cup Q_{v}^{\alpha}}$ and hence does not contain $p_{\alpha}$. By Theorem 1.1 this implies that $J\left(P_{\alpha}\right)$ is a solar Julia set which completes the proof of Theorem 1.2 .

\section{REFERENCES}

[BO06a] A. Blokh and L. Oversteegen, The Julia sets of quadratic Cremer polynomials, Topology and its Appl., 153 (2006), 3038-3050.

[BO06b] A. Blokh and L. Oversteegen, Monotone maps of Cremer Julia sets, preprint (2006).

[BC06] X. Buff, A. Chéritat, Quadratic Julia sets with positive area, preprint arXiv:math/0605514 2 (2008).

[BS94] S. Bullett and P. Sentenac, Ordered orbits of the shift, square roots, and the devill's staircase, Math. Proc. Camb. Phil. Soc. 115 (1994), pp. $451-481$.

[C01] A. Chéritat, Recherche densembles de Julia de mesure de Lebesgue positive, Thesis, Orsay (2001).

[CMR05] D. Childers, J. Mayer and J. Rogers, Jr., Indecomposable continua and the Julia sets of polynomials, II, Topology and its Applications 153 (2006), no. 10, 1593-1602.

[C05] D. Childers, Are there critical points on the boundaries of mother hedgehogs, to appear.

[DH85] A. Douady, J. H. Hubbard, Étude dynamique des polynômes complexes I, II Publications Mathématiques d'Orsay 84-02 (1984), 85-04 (1985).

[GMO99] J. Grispolakis, J. Mayer, and L Oversteegen, Building Blocks for Julia sets, Trans. Amer. Math. Soc. 351 (1999), no. 3, 1171-1201. 
[H96] J. Heath, Each locally one-to-one map from a continuum onto a tree-like continuum is a homeomorphism, Proc. Amer. Math. Soc. 124 (1996), 2571-2573.

[IS] H. Inou, M. Shishikura, The renormalization for parabolic fixed points and their perturbation, in preparation

[K00] J. Kiwi, Non-accessible critical points of Cremer polynomials, Erg. Th. and Dyn. Sys. 20 (2000), 1391-1403.

[K04] J. Kiwi, Real laminations and the topological dynamics of complex polynomials, Advances in Math. 184 (2004), no. 2, 207-267.

[LM97] M. Lyubich, Y. Minsky, Laminations in holomorphic dynamics, J. Differential Geom. 47 (1997), no. 1, 17-94.

[MR93] J. Mayer and J. Rogers, Jr., Indecomposable continua and the Julia sets of polynomials, Proc. Amer. Math. Soc. 117 (1993), no. 3, 795-802.

[Mn93] R. Mañé, On a theorem of Fatou, Bol. Soc. Bras. Mat. 24 (1993), 1-11.

[McM94] C. T. McMullen, Complex dynamics and renormalization, Annals of Mathematical Studies 135, Princeton University Press, Princeton, NJ (1994).

[McM98] C. T. McMullen, Self-similarity of Siegel disks and Hausdorff dimension of Julia sets, Acta Math., 180 (1998), pp. 247-292.

[Mil00] J. Milnor, Dynamics in one complex variable, 2nd edition, Vieweg, Wiesbaden (2000).

[Mun00] J. R. Munkres, Topology, 2nd edition, Prentice-Hall, Inc. (2000).

[Per94] R. Perez-Marco, Topology of Julia sets and hedgehogs, Publications Mathématiques d'Orsay 94-48 (1994).

[Per97] R. Perez-Marco, Fixed points and circle maps, Acta Math. 179 (1997), pp. 243-294.

[Pet96] C. L. Petersen, Local connectivity of some Julia sets containing a circle with an irrational rotation, Acta Math. 177 (1996), no. 2, pp. 163-224.

[Pom92] Ch. Pommerenke, Boundary behavior of conformal maps, SpringerVerlag, Berlin Heidelberg (1992).

[SZ99] D. Schleicher, S. Zakeri, On biaccessible points in the Julia set of a Cremer quadratic polynomial, Proc. Amer. Math. Soc. 128 (1999), pp. 933-937.

[Sor98] D. Sørensen, Describing quadratic Cremer polynomials by parabolic perturbations, Erg. Th. and Dyn. Sys., 18 (1998), pp. 739-758.

[Sul83] D. Sullivan, Conformal dynamical systems, Springer Lecture Notes in Mathematics, 1007 (1983), 725-752.

[Yoc95] J. C. Yoccoz, Petits diviseurs en dimension 1, Asterisque 231 (1995).

[Zak00] S. Zakeri, Biaccessibility in quadratic Julia sets, Erg. Th. and Dyn. Sys., 20 (2000), pp. 1859-1883.

(A. Blokh and L. Oversteegen) Department of Mathematics, University of Alabama at Birmingham, Birmingham, AL 35294-1170, USA 
(X. Buff and A. Chéritat) Institut de Mathématiques, Université Paul Sabatier, 118 route de Narbonne, 31062 Toulouse, France

E-mail address, A. Blokh: ablokh@math.uab.edu

E-mail address, L. Oversteegen: overstee@math.uab.edu

E-mail address, A. Chéritat: cheritat@picard.ups-tlse.fr

E-mail address, X. Buff: buff@picard.ups-tlse.fr 